\title{
Volatile Addition to the Inner Solar System Between 4.566 and 4.564 Ga: Evidence from Angrite Meteorites
}

\author{
A. R. Sarafian ${ }^{1}$, S. G. Nielsen ${ }^{1}$, H. R. Marschall ${ }^{1}$, G. A. Gaetani ${ }^{1}$, E. H. Hauri ${ }^{2}$, K. Righter ${ }^{3}$, E. Sarafian ${ }^{1}$ \\ 1. Woods Hole Oceanographic Institution, 266 Woods Hole Rd. Woods Hole MA \\ 2. Department of Terrestrial Magnetism, Carnegie Institution of Washington, Washington, DC \\ 3. NASA-JSC, Mailcode XI2, 2101 NASA Pkwy, Houston, TX
}

Water plays a fundamental role in planetary processes and is essential for the habitability of planets. Determining when and how the inner solar system received its water is critical in determining how planets evolved. The inner solar system planets are thought to have first accreted dry, then accreted wet material [1]. Recently, water has been found in eucrite phosphates [2], which crystallized at least by 815 million years after the start of the solar system [3], as defined by the age of CAI (4.567 Ga). Eucrites have an earth-like $\mathrm{H}, \mathrm{N}$, and $\mathrm{C}$ isotope signature [2], thus, probably accreted the same water source as Earth. The discovery of earth-like water in eucrites moves back the time of known water accretion to that of planetesimal formation in the inner solar system by more than 100 Myr. The oldest basaltic meteorites, angrites, can expand on recent work because some are older than eucrites and can help constrain the amount of early water accretion.

Angrites are a small group of differentiated meteorites that can be classified as intrusive and extrusive. They are extremely depleted in volatile metals, but the depletion is not seen in all elements, e.g., noble gasses [4]. Hydrogen, carbon, fluorine, and chlorine, volatile elements, have not been previously measured in angrites, and could help to constrain the timing, flux, and origin of volatile elements in the inner solar system.

SEM. We used the Hitachi TM3000 at Woods Hole Oceanographic Institution (WHOI) and the JEOL 7600F at NASA-Johnson Space Center to map all angrites prior to SIMS analysis.

EPMA. We used the Cameca SX100 at NASA Johnson Space Center, Houston, TX. An accelerating voltage of $20 \mathrm{kV}$ and a probe current of $40 \mathrm{nA}$ was used to measure major and minor element concentrations in olivine and pyroxene

SIMS. We used the Cameca IMS $6 f$ at the Carnegie Institution of Washington to measure $\mathrm{H}, \mathrm{C}, \mathrm{F}, \mathrm{Cl}$ and $\mathrm{S}$ in olivine and pyroxene. A $15 \mathrm{nA}$ primary beam was rastered over a $20 \times 20 \mu \mathrm{m}^{2}$ area and the central $\sim 4 \mathrm{x} 4 \mathrm{~m}^{2}$ of the secondary beam was collected using a field aperture.

We measured major, minor, and volatile, element concentrations in olivine in the angrites D'Orbigny and Sahara 99555. Angrite olivine water contents range from 7-25 $\mu \mathrm{g} / \mathrm{g} \mathrm{H}_{2} \mathrm{O}$ and strongly correlate with major element, $\mathrm{C}, \mathrm{F}$, and $\mathrm{Cl}$ contents.

To determine if post-crystallization diffusion occurred in angritic olivines, an empirical forward fractional crystallization model based on published mineral-melt equilibrium was constructed. The model reproduces the observed trends in major and volatile elements, which demonstrates that little to no post-crystallization modification occurred. We determined the volatile content of the primitive melt based on well-established olivine-melt partition coefficients. A calculation of the volatile content of the angrite mantle assuming 10-20\% partial melting, yields a mantle volatile content similar to the Earth's depleted upper mantle for $\mathrm{H}$ and $\mathrm{C}$, but a depletion in $\mathrm{F}$ and $\mathrm{Cl}$. Even though angrites are the most volatile element-depleted basalts, exhibited by an extremely low Na content ( $\mathrm{Na}$ Angrites $=20 \mu \mathrm{g} / \mathrm{g}$, 
Moon $=900 \mu \mathrm{g} / \mathrm{g}$, Earth $=2700 \mu \mathrm{g} / \mathrm{g}$, chondrites $=4000-7000 \mu \mathrm{g} / \mathrm{g}$, the $\mathrm{C} / \mathrm{H}, \mathrm{F} / \mathrm{H}, \mathrm{Cl} / \mathrm{H}$ of angrites are nearly identical to carbonaceous chondrites, suggesting a carbonaceous-chondrite source of these volatile elements.

The timing of carbonaceous chondritic addition to the inner solar system is debated [5]. The geologic constrains of angrites provide a robust time window of water addition to the inner solar system. Addition of chondritic material to the angrite parent body occurred after core formation, which happened between 0.5 to 2 Myr after CAIs [6]. The oldest angrites have a crystallization age of 3.4-4.0 Myr after CAIs [8]. We suggest that post core-formation, there was an addition of chondrite material [7], that was addition of carbonaceous chondrite material. Mixing calculations show the addition of $<1 \%$ of carbonaceous chondrite to the angrite parent body between core formation (0.5-2 Myr after CAIs) and crystallization of the basalts (3.5-4.0 Myr after CAIs). We therefore provide a robust constraint on when and how much water and other volatile elements were accreted to the inner solar system within the first few million years of solar system history.

The recently proposed Grand-Tack model predicts an inward scattering of volatile-rich planetesimals and debris from the outer solar system, where carbonaceous chondrites may have formed. Hence, the Grand Tack model is a possible mechanism by which volatiles could have been delivered to the APB very early in solar system history. With our added constraints, the Grand Tack must have initiated no later than $4564 \mathrm{Ma}$. In addition, the Grand Tack model requires that gas was still present in the nebular disc during Jupiter's migration and that the migration terminated as the nebular gas dissipated. Chondrules, a common constituent of chondrite meteorites, are thought to have formed in the presence of nebular gas. Hence, if the Grand Tack was indeed the physical mechanism by which volatiles were brought into the inner solar system, then no chondrules should be younger than $4563 \mathrm{Ma}$, which is in agreement with the most recent chondrule chronology [9]. Our data is consistent with previous estimates of the source of water for the inner solar system [2]. Here we report the flux of water to the inner solar system and provide a time constraint on a plausible mechanism that may have hydrated the inner solar system.

\section{References:}

[1] Schönbächler, M., et al., Science, 2010. 328(5980): p. 884-887.

[2] Sarafian, A.R., et al., Science, 2014. 346(6209): p. 623-626.

[3] Touboul, M., et al., Geochimica et Cosmochimica Acta, 2015. 156: p. 106-121.

[4] Busemann, H., S. Lorenzetti, and O. Eugster, Geochimica et cosmochimica acta, 2006. 70(21): p. 5403-5425.

[5] Barnes, J., et al., Chemical Geology, 2012. 337-338: p. 48-55.

[6] Kleine, T., et al., Geochimica et Cosmochimica Acta, 2012. 84: p. 186-203.

[7] Riches, A.J., et al., Earth and Planetary Science Letters, 2012. 353: p. 208-218.

[8] Schiller, M., et al., Earth and Planetary Science Letters, 2015. 420: p. 45-54.

[9] Connelly, J.N., et al., Science, 2012. 338(6107): p. 651-655. 